\title{
Fluid-retention side-effects of the chemotherapy-supportive treatment interleukin-11: mathematical modelling of putative underlying mechanisms
}

\author{
YURI KHEIFETZ $\dagger$, MORAN ELISHMERENI $\dagger$, SMADAR HOROWITZ $\dagger$ and ZVIA AGUR $\dagger{ }^{*}$ \\ $\dagger$ Institute for Medical Biomathematics (IMBM), Bene-Ataroth, Israel \\ $\ddagger$ Optimata Ltd., Ramat Gan, Israel
}

(Received 31 May 2006; in final form 14 July 2006)

\begin{abstract}
Interleukin-11 (IL-11) is a pleiotropic thrombopoietic cytokine and immune modulator, clinically approved for alleviation of chemotherapy-induced thrombocytopenia in nonmyeloid malignancies. IL-11 therapy exerts fluid accumulation-associated adverse effects, complicating its administration and limiting its use. Implementation of standard biomathematical techniques to assess these effects is not possible, due to incomplete knowledge of the underlying mechanisms. This study investigates IL-11-induced blood volume expansion (BVE) by a new mathematical modelling methodology. Alternative models for BVE following IL-11 therapy were constructed, calibrated with clinical information and simulated in a number of treatment scenarios. The models demonstrated high compliance and were equally capable of reliably predicting BVE in a wide range of treatments, provided sufficient data. Model simulations indicate that frequent and low dose IL-11 regimens are favored for ensuring minimal fluid retention, upon the current IL-11 therapy.
\end{abstract}

Keywords: Blood volume expansion; Thrombocytopenia; Anti-inflammation therapy; Ordinary differential equations; Cytokine therapy; Incomplete inverse problem

AMS Subject Classification: 34A34; 93C10; 93C15; 93C40; 93C41; 93C57

\section{Introduction}

Cytokines and growth factors are increasingly being discovered and evaluated for therapeutic purposes and beneficial responses have been observed in a wide array of cancerous, inflammatory and autoimmune diseases [1,2]. However, many of these specifically targeted treatments lead to detrimental adverse effects, greatly limiting their clinical use. For example, interleukin-2, a critical immunoregulator applied in malignant diseases to enhance antitumor immunity, can exert vascular leak syndrome and immunopathologies such as thyroid disorders and rheumatoid arthritis [3,4]. Similarly, administration of interferon- $\alpha$ in viral and cancerous diseases has been associated with the exacerbation or occurrence of the autoimmune diseases thyroiditis and diabetes, as well as hematological disorders [4]. Developments in molecular and pharmaceutical drug design aim to enhance treatment

*Corresponding author. Tel.: +972-3-9733075. Fax: +972-3-9733410. Email: agur@imbm.org

Computational and Mathematical Methods in Medicine

ISSN 1748-670X print/ISSN 1748-6718 online (C) 2006 Taylor \& Francis

http://www.tandf.co.uk/journals

DOI: 10.1080/10273660600969018 
efficacy and resolve such conflicts. As these methods are time- and resource-consuming, new approaches are inevitably being considered.

Interleukin-11 (IL-11), a member of the gp130 family of cytokines, is a bone-marrowderived pleiotropic growth factor. Among its varied effects, IL-11 stimulates differentiation and maturation of thrombopoietic progenitors, inducing platelet production [5]. The genetically-engineered analog, recombinant-human-IL-11 (rhIL-11), was shown to elicit dose-dependent platelet increases in thrombocytopenic patients and was clinically approved for alleviation of myelosuppressive conditions [5-8]. Furthermore, IL-11 application has regenerative effects on the gastrointestinal epithelium, decreasing the inflammatory mediators tumor necrosis factor- $\alpha$, interleukin- $1 \beta$ and interferon- $\gamma$ [9]. However, IL-11 exerts multiple and varied side-effects, mainly associated with BVE: reversible low-grade edema and anemia on the one hand, and life-threatening arrhythmias on the other [10-12]. Accordingly, IL-11 therapy, being limited to patients with either severe thrombocytopenia, or with a very positive medical history, is currently unable to replace standard platelet transfusion as a supportive treatment for alleviating chemotherapy-induced toxicity [13].

To find treatment conditions that allow IL-11 therapy and yet minimize BVE, accurate quantification of this effect is required. Methods of biomathematical modelling can be applied for such purposes. Such models are used for analyzing effects of environmental disturbances on population survival, in general, and the effect of drug schedules on chemotherapy efficacy/toxicity ratio, in particular [15,14]. Mathematical models for thrombopoiesis have been developed for studying periodic thrombocytopenia [16]. Another recent mathematical model describing detailed thrombopoiesis was employed for optimizing treatment strategies of thrombopoietin. The qualitative and quantitative predictions of the models were prospectively validated in the preclinical setting [17]. Recent mathematical models of tumor-immune interactions emphasize the relative role of cytokines in anticancer responses and evaluate efficacy of immunotherapy in controlling tumor growth [18-20].

Since the mechanism underlying IL-11-mediated water accumulation is not clearly defined and data is limited [11,21], the current biomathematical approach to this phenomenon cannot be applied. Therefore, we replace this approach by a new method using multiple alternative models to describe BVE following IL-11 therapy. We show that, provided preliminary experimental data from various stages of therapy, the different models reliably predict dynamics of water retention within a certain span of IL-11-administration strategies. This allows us to understand the central factors influencing IL-11-induced BVE and to assess the clinical applicability of each of these IL-11 therapy schedules.

\section{Methods}

\subsection{Biological background and model structure}

The physiological regulation of body fluids is a well-controlled and stable system [22,23]. Thus, we assume that any administration of IL-11 is a perturbation in this system, which ultimately returns to steady state. With this assumption, the modelling of BVE effects may not require inclusion of all existing biological factors in the process. We assume the contrary: key elements and simple feedback forces, entailing a minimal number of degrees of freedom, suffice for a biologically-based mathematical description.

As the IL-11-associated BVE mechanism has not been fully established, we refrain from applying a conservative single mathematical model to describe the discussed process. Instead, a method of multiple modelling [24] is used, based on the following assumption: by describing 
IL-11-induced BVE through several models, each relying on alternative mechanisms but all producing the same behaviour, it is possible to faithfully depict this effect, despite the obscurity of the actual mechanism. We postulate that this ability is further increased in our setting, as the space of models that capture the behaviour of stable systems is assumed to be relatively narrow [24]. Furthermore, by employing BVE measurements to evaluate internal parameters, we consider this system as an incomplete inverse mathematical problem.

Accordingly, a family of three closely-related biologically-based ordinary differential equation (ODE) models are formed, each testing a different pathway of IL-11 therapyaffected BVE. Basic water homeostasis mechanisms, essential volume properties and established endocrinal feedback effects [23,25], together with an IL-11-induced perturbation of the system, are incorporated in each model. An iterative process of model calibration and comparison, elaborated in a previous work [24], enables us to evaluate the similarity of the models and consequently, their robustness in predicting BVE dynamics.

Water accumulation due to IL-11 administration has been postulated to occur via several possible pathways. Initial peripheral vasodilation of blood vessels, induced by the drug, may be followed by a renal compensatory volume intake, endocrinally aided. Alternatively, an effect of the cytokine on the kidneys, either by invoking hormonal up-regulation, or by modifying sodium absorption channels, can lead directly to fluid elevation [11]. Thus, the IL11 effect can be described through an influence on vessel dynamics (introduced in model 1), or, more directly, through immediate elevation of volume (introduced in model 2 and model 3).

Endocrinal regulation on vascular and volume entities consists mainly of the reninangiotensin-aldosterone system (RAAS), counter-regulated by the atrial natriuretic peptide (ANP) and associated factors. The RAAS is stimulated following low blood pressure and subsequently promotes vasoconstriction and volume retention, while ANP is secreted as a result of high blood pressure and acts to induce vasodilation and increased diuresis [23]. Hence, we depict the general effects of these opposing systems in a manner by which both vessel surface and volume absorption are regulated by each of these factors. To further differentiate between the models, model 1 and model 2 consist of a full endocrinal feedback effect (exerted on both the vessel and volume compartments), while model 3 contains a partial feedback effect (exerted on blood volume only).

To describe IL-11-induced retention of body waters, several time scales are taken into account in the models. This coincides with well-known biological effects; neuronal feedbacks to hemodynamic changes are fast, occurring within minutes, while endocrinal responses are slower but still within the time frame of hours-days, and in disease, weeks. Conversely, adaptation of mechanistic and structural components of the organism to chronic water-pressure modifications requires months-years [23]. As the latter effect is less relevant in the context of short-term IL-11 therapy, the models take into account only the time span of minutes-weeks.

With respect to the above considerations, the following observables comprise the models: (A) IL-11 concentration in the tissue of administration; (B) plasma IL-11 concentration; (C) average surface of blood vessels; (D) blood pressure; (E) blood volume; (F) endocrinal factors up-regulating blood vessel structure and blood volume; (G) endocrinal factors downregulating blood vessel structure and blood volume. All equations are a function of time, initiated at $t=0$. The corresponding equations are elaborated below.

\subsection{Model equations}

Three different models, which vary in (i) the target for IL-11 effect and (ii) the target for the feedback hormonal effect, are represented. Model 1 assumes IL-11-induced blood vessel 
dilation and full endocrinal function; model 2 assumes IL-11-induced volume expansion and full endocrinal function; and model 3 assumes IL-11 induced volume expansion and partial endocrinal function. The models are identical in all other assumptions, as described below.

2.2.1 IL-11 dynamics. Since IL-11 is applied subcutaneously [5], dynamics are represented by a one-compartment pharmacokinetic model, with first-order absorption from the external tissue [26]. The IL-11 concentrations in the tissue $\left(I_{\mathrm{T}}\right)$ and plasma $\left(I_{\mathrm{P}}\right)$ are given by

$$
\dot{I}_{\mathrm{T}}=-c_{1} I_{\mathrm{T}},
$$

and

$$
\dot{I}_{\mathrm{P}}=f c_{1} I_{\mathrm{T}}-c_{2} I_{\mathrm{P}}
$$

where the initial conditions are $I_{\mathrm{T}}(t=0)=I_{\text {dose }}, I_{\text {dose }}$ denoting the administered IL-11 concentration and $I_{\mathrm{P}}(t=0)=0$. The coefficient $c_{1}$ denotes the rate of drug transport from the primary tissue to the plasma, $c_{2}$ is the drug clearance rate, and the fraction of transferred drug, an indicator of bioavailability, is $f$.

IL-11 kinetics were analytically solved. For a single injection, equation (1) becomes

$$
I_{\mathrm{T}}(t)=I_{\mathrm{T}}(0) \mathrm{e}^{-c_{1} t} .
$$

To generalize IL-11 therapy to include a long-term multiple administrations [6,7], the standard pharmacokinetic transformation is used. Thus, for a strategy of equally distributed and constant intensity administrations, equation (1) is

$$
I_{\mathrm{T}}(t)=\frac{I_{\mathrm{T}}(0)\left(\mathrm{e}^{-c_{1}(t-h[t / h])}\right)\left(1-\mathrm{e}^{-h c_{1}([t / h]+1)}\right)}{\left(1-\mathrm{e}^{-h c_{1}}\right)}
$$

where $h$ is the time between consecutive injections.

2.2.2 Blood pressure dynamics. We describe blood pressure by its classical interpretation, as determined by heart rate, stroke volume (blood ejected by the heart with each contraction) and the resistance of the blood vessels to blood flow (systemic vascular resistance) [25]. For simplicity, we assume (i) no significant heart rate variability, as clinically observed [11], and (ii) an inverse correlation between vascular resistance and vascular capacity. Accordingly, blood pressure can be a function of blood volume and the vascular capacity (expressed in terms of vessel surface), as described in the following equation:

$$
P(t)=\left(\frac{V(t)}{S(t)^{3 / 2}}\right)^{n_{0}}
$$

The function $P(t)$ is blood pressure at time $t, S(t)$ denotes overall vessel surface at time $t, V(t)$ is blood volume at time $t$ and $n_{0}$ is a parameter. Steady state values of pressure, overall vessels surface and volume are given by $P_{\mathrm{st}}, S_{\mathrm{st}}$ and $V_{\mathrm{st}}$, respectively.

2.2.3 Endocrinal feedback dynamics. Dynamics of the compensatory RAAS-resembling effects that elevate blood pressure $\left(H_{\mathrm{U}}\right)$ and the down-regulating, compensatory ANP-resembling 
effects $\left(H_{\mathrm{D}}\right)$ are given by

$$
\begin{aligned}
& \dot{H}_{\mathrm{U}}=\alpha_{1} g\left(P_{\mathrm{st}}-P(t)\right)-d_{1} H_{\mathrm{U}}(t), \\
& \dot{H}_{\mathrm{D}}=\alpha_{2} g\left(P(t)-P_{\mathrm{st}}\right)-d_{2} H_{\mathrm{D}}(t) .
\end{aligned}
$$

In equations (6) and (7), formation rates $\left(\alpha_{1}, \alpha_{2}\right)$ and degradation rates $\left(d_{1}, d_{2}\right)$ of the endocrinal effects are taken as constants. Blood pressure deviations allow formation of each factor, using the function:

$$
g(x)= \begin{cases}x & \text { if } x>0 \\ 0 & \text { if } x \leq 0\end{cases}
$$

It is noted that these depictions are general and do not refer to hormonal concentrations per se, but rather, to effects associated with endocrinal deviations.

2.2.4 Blood vessel dynamics. In our model, the vascular structure relates to the average surface of the inner endothelium layer of the blood vessels, in two-dimensional units. IL-11induced vessel surface enlargement (in model 1) and $H_{\mathrm{U}^{-}}$-induced vasoconstriction or $H_{\mathrm{D}^{-}}$ induced vasodilation (in models 1-2), are described by biologically-compatible sigmoidal functions. Model 3 does not enable vascular resizing in response to IL-11 or hormones. Additionally, all three models incorporate a simple intrinsic feedback effect to vessel steady state $\left(S_{\mathrm{st}}\right)$ in the blood vessel dynamics. Thus, the equation for model 1 is

$$
\dot{S}=\frac{\beta_{0}\left(I_{\mathrm{P}}(t)\right)^{r_{0}}}{k_{0}+\left(I_{\mathrm{P}}(t)\right)^{r_{0}}}-\frac{\beta_{1}\left(H_{\mathrm{U}}(t)\right)^{r_{1}}}{k_{1}+\left(H_{\mathrm{U}}(t)\right)^{r_{1}}}+\frac{\beta_{2}\left(H_{\mathrm{D}}(t)\right)^{r_{2}}}{k_{2}+\left(H_{\mathrm{D}}(t)\right)^{r_{2}}}+\beta_{3}\left(S_{\mathrm{st}}-S(t)\right),
$$

The equations for model 2 and model 3 are respectively:

$$
\begin{gathered}
\dot{S}=-\frac{\beta_{1}\left(H_{\mathrm{U}}(t)\right)^{r_{1}}}{k_{1}+\left(H_{\mathrm{U}}(t)\right)^{r_{1}}}+\frac{\beta_{2}\left(H_{\mathrm{D}}(t)\right)^{r_{2}}}{k_{2}+\left(H_{\mathrm{D}}(t)\right)^{r_{2}}}+\beta_{3}\left(S_{\mathrm{st}}-S(t)\right), \\
\dot{S}=\beta_{3}\left(S_{\mathrm{st}}-S(t)\right),
\end{gathered}
$$

where $k_{0}, k_{1}, k_{2}$ and $r_{0}, r_{1}, r_{2}$ are positive parameters. Vascular modification rates in response to IL-11, $H_{\mathrm{U}}$ and $H_{\mathrm{D}}$ are denoted as $\beta_{0}, \beta_{1}$ and $\beta_{2}$, respectively, and $\beta_{3}$ is the coefficient of direct vessel surface regulation. As mentioned above, effects of vascular hypertrophy and remodelling are neglected, as such effects are observed in more chronic hemodynamic irregularities and larger time scales [25].

2.2.5 Blood volume dynamics. Volume dynamics can initially be characterized by water absorption-excretion differences,

$$
\dot{V}=A(t)-E(t),
$$

where $A$ is the function of volume absorbtion and $E$ is the volume excretion function. Though in reality sharp changes in either rates may exist, these effects are neglected and parameters are assumed to be continuous. Absorption is simplified in our model to be constant at all 
times, evaluated at a steady state volume $A(t)=A_{\mathrm{st}}$. In contrast, the function of excretion in our model is not constant, but susceptible to (i) systemic blood pressure deviations, known to specifically act on renal water excretion, and to (ii) the drug-induced effects. The function is therefore set to satisfy a pressure-related difference between normal steady state excretion $\left(E_{\mathrm{st}}\right)$ and excretion in the IL-11 scenario $\left(E_{\text {drug }}\right)$ :

$$
E(t)=\left(\frac{P(t)}{P_{\mathrm{st}}}\right)^{n_{1}}\left(E_{\mathrm{st}}-E_{\mathrm{drug}}(t)\right)
$$

Parameter $n_{1}$ is the power coefficient of the function. Under these terms, volume dynamics may be described by

$$
\dot{V}=A_{\mathrm{st}}-\left(\frac{P(t)}{P_{\mathrm{st}}}\right)^{n_{1}}\left(E_{\mathrm{st}}-E_{\mathrm{drug}}(t)\right) \text {. }
$$

However, if we assume equal absorption and excretion rates $\left(A_{\mathrm{st}}=E_{\mathrm{st}}\right)$ under normal conditions, the volume dynamics can be formed as

$$
\dot{V}=E_{\mathrm{st}}\left(1-\left(\frac{P(t)}{P_{\mathrm{st}}}\right)^{n_{1}}\right)+\left(\frac{P(t)}{P_{\mathrm{st}}}\right)^{n_{1}}\left(E_{\mathrm{drug}}(t)\right) .
$$

As previously described, in all three models, the term $E_{\text {drug }}$ is assumed to be affected by the hormones, whereas in models 2-3 only, it is taken as affected by IL-11 as well. As in the description of blood vessels, both the IL-11 influence (models 2-3) and the endocrinal influence (models 1-3) on volume dynamics are non-linear. Thus, for model 1 we have

$$
E_{\mathrm{drug}}(t)=\frac{\gamma_{1}\left(H_{\mathrm{U}}(t)\right)^{q_{1}}}{m_{1}+\left(H_{\mathrm{U}}(t)\right)^{q_{1}}}-\frac{\gamma_{2}\left(H_{\mathrm{D}}(t)\right)^{q_{2}}}{m_{2}+\left(H_{\mathrm{D}}(t)\right)^{q_{2}}}
$$

and for models 2-3, we have

$$
E_{\text {drug }}(t)=\frac{\gamma_{0}\left(I_{\mathrm{P}}(t)\right)^{q_{0}}}{m_{0}+\left(I_{\mathrm{P}}(t)\right)^{q_{0}}}+\frac{\gamma_{1}\left(H_{\mathrm{U}}(t)\right)^{q_{1}}}{m_{1}+\left(H_{\mathrm{U}}(t)\right)^{q_{1}}}-\frac{\gamma_{2}\left(H_{\mathrm{D}}(t)\right)^{q_{2}}}{m_{2}+\left(H_{\mathrm{D}}(t)\right)^{q_{2}}},
$$

where $m_{0}, m_{1}, m_{2}$ and $q_{0}, q_{1}, q_{2}$ are positive constants. The IL-11, $H_{\mathrm{U}}$ and $H_{\mathrm{D}}$-afflicted rates of water excretion are denoted $\gamma_{0}, \gamma_{1}$ and $\gamma_{2}$, respectively. Substituting equation (15) by equations (16) -(17), the final volume dynamics for model 1 are given by

$$
\dot{V}=E_{\mathrm{st}}\left(1-\left(\frac{P(t)}{P_{\mathrm{st}}}\right)^{n_{1}}\right)+\left(\frac{P(t)}{P_{\mathrm{st}}}\right)^{n_{1}} \times\left(\frac{\gamma_{1}\left(H_{\mathrm{U}}(t)\right)^{q_{1}}}{m_{1}+\left(H_{\mathrm{U}}(t)\right)^{q_{1}}}-\frac{\gamma_{2}\left(H_{\mathrm{D}}(t)\right)^{q_{2}}}{m_{2}+\left(H_{\mathrm{D}}(t)\right)^{q_{2}}}\right)
$$

and for model 2-3 are given by

$$
\begin{aligned}
\dot{V}= & E_{\mathrm{st}}\left(1-\left(\frac{P(t)}{P_{\mathrm{st}}}\right)^{n_{1}}\right) \\
& +\left(\frac{P(t)}{P_{\mathrm{st}}}\right)^{n_{1}} \times\left(\frac{\gamma_{0}\left(I_{\mathrm{P}}(t)\right)^{q_{0}}}{m_{0}+\left(I_{\mathrm{P}}(t)\right)^{q_{0}}}+\frac{\gamma_{1}\left(H_{\mathrm{U}}(t)\right)^{q_{1}}}{m_{1}+\left(H_{\mathrm{U}}(t)\right)^{q_{1}}}-\frac{\gamma_{2}\left(H_{\mathrm{D}}(t)\right)^{q_{2}}}{m_{2}+\left(H_{\mathrm{D}}(t)\right)^{q_{2}}}\right) .
\end{aligned}
$$




\subsection{Model calibration}

All three models were calibrated to experimental data with various initial conditions. Customized curve-fitting methods and local search were implemented in Matlab and $\mathrm{C}++$ programming for this purpose. For initial model calibration, data of hemoglobin concentrations $(\mathrm{g} / \mathrm{dl})$, measured during one week of IL-11 therapy (7 daily doses of $25 \mu \mathrm{g} / \mathrm{kg}$ ), were taken from a previous study in healthy humans [11] and adapted to measurements of blood volume (liters). The conversion was enabled by considering a unified initial steady state blood volume $\left(V_{\mathrm{st}}\right)$ of 5.5 liters, that corresponds to a unified mean hemoglobin level of $14.5 \mathrm{~g} / \mathrm{dl}$. Models approximated within the standard deviations of this experimental data set, denoted $D E$, were considered equivalent. The majority of parameters were evaluated, yet some were normalized to 1 (table 1). An insufficient amount of data points in $D E$ led us to examine different sizes of data sets. Thus, for refined calibration of the model, four homogenously-distributed data sets, denoted $D 1-D 4$ and with increasing sizes, were obtained from model 1 simulations under the experimental treatment regimen, as previously described in Ref. [24].

Table 1. Model parameters, as yielded by curve-fitting of the three models to experimental data [11].

\begin{tabular}{|c|c|c|c|c|}
\hline Parameter & Definition & Model 1 & Model 2 & Model 3 \\
\hline$I_{\text {dose }}$ & Administered IL-11 concentration & 1 & 1 & 1 \\
\hline$V_{\text {st }}$ & Steady state blood volume & 1 & 1 & 1 \\
\hline$P_{\mathrm{st}}$ & Steady state blood pressure & 1 & 1 & 1 \\
\hline$S_{\text {st }}$ & Steady state blood vessel surface & 1 & 1 & 1 \\
\hline$E_{\text {st }}$ & Steady state volume excretion rate & 0.01 & 0.01 & 0.01 \\
\hline$c_{1}$ & Transfer rate of IL-11 from tissue to plasma & 0.018 & 0.025 & $10^{-7}$ \\
\hline$c_{2}$ & Clearance rate of IL-11 & 0.676 & 0.186 & 1.041 \\
\hline$f$ & Fraction of absorbed IL-11 in plasma & 0.28 & 0.03 & 0.13 \\
\hline$n_{0}$ & Power coefficient of $V / S$ fraction & 3.135 & 1.027 & 1.011 \\
\hline$n_{1}$ & Power coefficient of $E$ dependence on blood pressure & 1.154 & 1.077 & 1 \\
\hline$\alpha_{1}$ & Formation rate of $H_{\mathrm{U}}$ effect & 0.07 & 1.312 & 0.139 \\
\hline$\alpha_{2}$ & Formation rate of $H_{\mathrm{D}}$ effect & 0.208 & 0.125 & 0.027 \\
\hline$d_{1}$ & Degradation rate of $H_{\mathrm{U}}$ effect & 0.006 & 0.013 & 0.001 \\
\hline$d_{2}$ & Degradation rate of $H_{\mathrm{D}}$ effect & 0.008 & 0.021 & 0.001 \\
\hline$\beta_{0}$ & Coefficient of IL-11 effect on blood vessels & 0.19 & 0.226 & 0.185 \\
\hline$\beta_{1}$ & Coefficient of $H_{\mathrm{U}}$ effect on blood vessels & 0.016 & 0.001 & 0.1 \\
\hline$\beta_{2}$ & Coefficient of $H_{\mathrm{D}}$ effect on blood vessels & 0.505 & 0.47 & 0.165 \\
\hline$\beta_{3}$ & Rate of blood vessel deviation from steady state & 0.975 & 0.001 & 0.72 \\
\hline$k_{0}$ & Steepness coefficient of IL-11 effect on blood vessels & 2.75 & 2.89 & 1 \\
\hline$k_{1}$ & Steepness coefficient of $H_{\mathrm{U}}$ effect on blood vessels & 0.017 & 9.32 & 0.89 \\
\hline$k_{2}$ & Steepness coefficient of $H_{\mathrm{D}}$ effect on blood vessels & 32.57 & 38.69 & 1 \\
\hline$r_{0}$ & Power coefficient of IL-11 effect on blood vessels & 1.425 & 1.25 & 1.83 \\
\hline$r_{1}$ & Power coefficient of $H_{\mathrm{U}}$ effect on blood vessels & 5.06 & 7.83 & 1.5 \\
\hline$r_{2}$ & Power coefficient of $H_{\mathrm{D}}$ effect on blood vessels & 4.99 & 4.30 & 1.56 \\
\hline$\gamma_{0}$ & Coefficient of IL-11 effect on blood volume & 0.19 & 0.226 & 0.185 \\
\hline$\gamma_{1}$ & Coefficient of $H_{\mathrm{U}}$ effect on blood volume & 0.346 & 1.55 & 0.332 \\
\hline$\gamma_{2}$ & Coefficient of $H_{\mathrm{D}}$ effect on blood volume & 0.39 & 0.38 & 0.214 \\
\hline$m_{0}$ & Steepness coefficient of IL-11 effect on blood volume & 2.75 & 2.89 & 1 \\
\hline$m_{1}$ & Steepness coefficient of $H_{\mathrm{U}}$ effect on blood volume & 1 & 1 & 1 \\
\hline$m_{2}$ & Steepness coefficient of $H_{\mathrm{D}}$ effect on blood volume & 1 & 1 & 1 \\
\hline$q_{0}$ & Power coefficient of IL-11 effect on blood volume & 1.425 & 1.25 & 1.83 \\
\hline$q_{1}$ & Power coefficient of $H_{\mathrm{U}}$ effect on blood volume & 1.55 & 3.15 & 1.39 \\
\hline$q_{2}$ & Power coefficient of $H_{\mathrm{D}}$ effect on blood volume & 3.13 & 1.02 & 1.011 \\
\hline
\end{tabular}




\subsection{Model simulations and comparison}

Models were simulated under a wide spectrum of treatment schedules (table 2) in Matlab and $\mathrm{C}++$ programming. Resulting blood volume and pressure dynamics were compared between the different models, following (i) initial calibration with experimental data and (ii) refined calibration with four expanded data sets. Comparisons of calculated blood volumes were accomplished by (i) simple qualitative evaluation of volume dynamics and by (ii) different similarity indexes (hereafter SI), minimal values of which indicate strong compatibility between the models. We computed SIs of four types and evaluated predictability for each treatment schedule (table 2), as described in Ref. [24].

\section{Results}

The predictability of the differently-formed models of IL-11-stimulated BVE depends upon their ability to generate similar dynamics. Thus, their calibration on an experimental data set $(D E)$ was called for. In multiple attempts with a wide array of initial parameter values, each of the models was approximated to $D E$ (figure 1). Rational parameters (table 1) were yielded, rendering the models equally relevant for simulations. To test whether the models globally yielded the same behaviour, the three models were simulated under the experimental schedule of seven $25 \mu \mathrm{g} / \mathrm{kg} /$ day IL-11 injections (table 2, treatment 0 ) with parameter values from different successful fits. The three models showed diverse results, both within the therapeutic window and outside it. Specifically for the post therapeutic time, model outputs were vastly incomparable, as indicated by the different steady states (figure 2). Only model 1 produced BVE dynamics which ultimately returned to steady state. We concluded that calibration according to $D E$ did not suffice to give unified dynamics and models 1-3 were not dependable for reliable generation of BVE behaviour.

We then investigated the possibility that curve-fitting to a wider data set permits higher compliance between the model's BVE outputs. In the absence of reliable clinical information, differently-sized data sets $(D 1-D 4)$ were derived from the output of model 1

Table 2. Characteristics and predictability marks of simulated IL-11 treatment schedules. The original experimental schedule (control) and four groups of additional treatments (1-11), each categorized by the dose intensity, inter-dosing interval, treatment duration and drug administration rate, were simulated. Predictability marks, calculated (as described in Methods and Ref. [24]) for the model's comparison following calibration with a 3.5 -fold incremented model 1-derived data set, were assigned to each treatment. A, no predictability; B, low predictability; C, average predictability; and $\mathrm{D}$, good predictability.

\begin{tabular}{|c|c|c|c|c|c|c|}
\hline $\begin{array}{l}\text { Treatment } \\
\text { group }\end{array}$ & $\begin{array}{l}\text { Treatment } \\
\text { (no.) }\end{array}$ & $\begin{array}{c}\text { Dose } \\
(\mu \mathrm{g} / \mathrm{kg})\end{array}$ & $\begin{array}{l}\text { Inter-dosing } \\
\text { interval }(\mathrm{h})\end{array}$ & $\begin{array}{l}\text { Duration } \\
\text { (days) }\end{array}$ & $\begin{array}{c}\text { Adm. rate } \\
(\mu \mathrm{g} / \mathrm{kg} / \text { day })\end{array}$ & $\begin{array}{l}\text { Predictability } \\
\text { grade }\end{array}$ \\
\hline Control (exp.) & 0 & 25 & 24 & 8 & 25 & $\mathrm{D}$ \\
\hline \multirow[t]{2}{*}{ Prolonged } & 1 & 25 & 24 & 30 & 25 & $\mathrm{D}$ \\
\hline & 2 & 20 & 24 & 30 & 20 & $\mathrm{D}$ \\
\hline \multirow[t]{4}{*}{ High frequency } & 3 & 3 & 5 & 8 & 14.4 & $\mathrm{~B}$ \\
\hline & 4 & 5 & 5 & 8 & 24 & $\mathrm{D}$ \\
\hline & 5 & 7.5 & 5 & 8 & 36 & B \\
\hline & 6 & 12.5 & 5 & 8 & 60 & A \\
\hline \multirow[t]{2}{*}{ Low frequency } & 7 & 32.5 & 50 & 20 & 15.6 & $\mathrm{C}$ \\
\hline & 8 & 50 & 50 & 20 & 24 & $\mathrm{D}$ \\
\hline \multirow[t]{3}{*}{ Random periodicity } & 9 & 25 & Random & 30 & $\sim 16$ & $\mathrm{C}$ \\
\hline & 10 & 37.5 & Random & 30 & $\sim 24$ & $\mathrm{D}$ \\
\hline & 11 & 50 & Random & 30 & $\sim 32$ & B \\
\hline
\end{tabular}




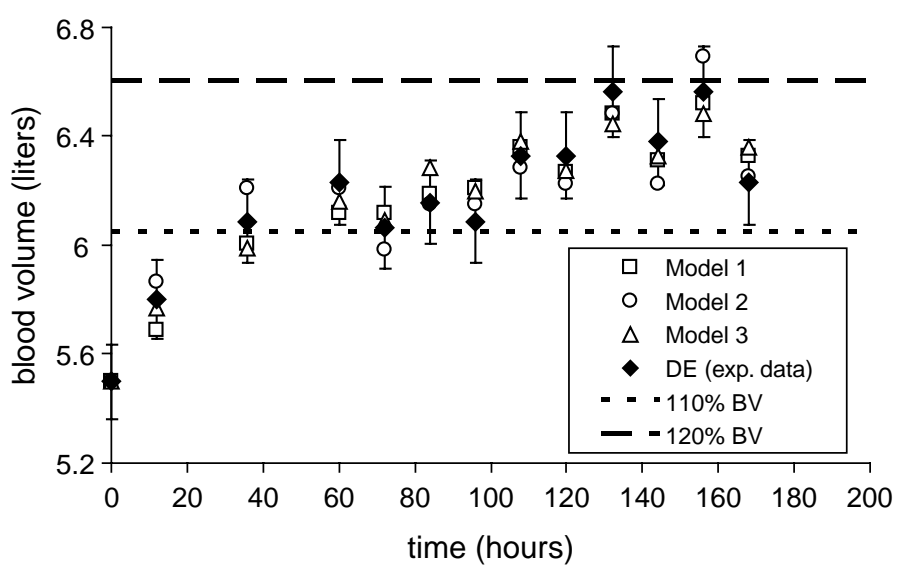

Figure 1. Calibration of alternative IL-11-induced BVE models with clinical experimental data $(D E)$. Blood volume levels (in liters), derived from simulations of models 1, 2 and 3 following curve-fitting to data from a previous experiment in healthy adults [11], are shown relative to experimental points. About 10 and $20 \%$ elevations in blood volume are shown for reference.

(figure 2), which was assumed most realistic, since body water is stringently regulated and should resume steady state. These data were taken from stages both within therapy and following treatment termination. Contrary to the previous settings, after $D 1$ calibration, the models complied to form unified dynamics (figure 3(A)) under treatment 0 . Calibration with $D 2, D 3$ (data not shown), or $D 4$ (figure 3(B)) further improved the models' compliance. A data size-dependent enhanced similarity of the models was confirmed: Declining SI values, indicating lower differences between the models, were seen when using more data (figure 3(C)), resulting in enhanced predictability of the models due to their robust compliance. Notwithstanding, a possible saturation in this effect was noticed in the decelerated differences towards the highest data sizes, implying the existence of a threshold. Collectively, the three models are equally able to reliably describe BVE dynamics under treatment 0 (table 2), given sufficient and well-distributed experimental data.

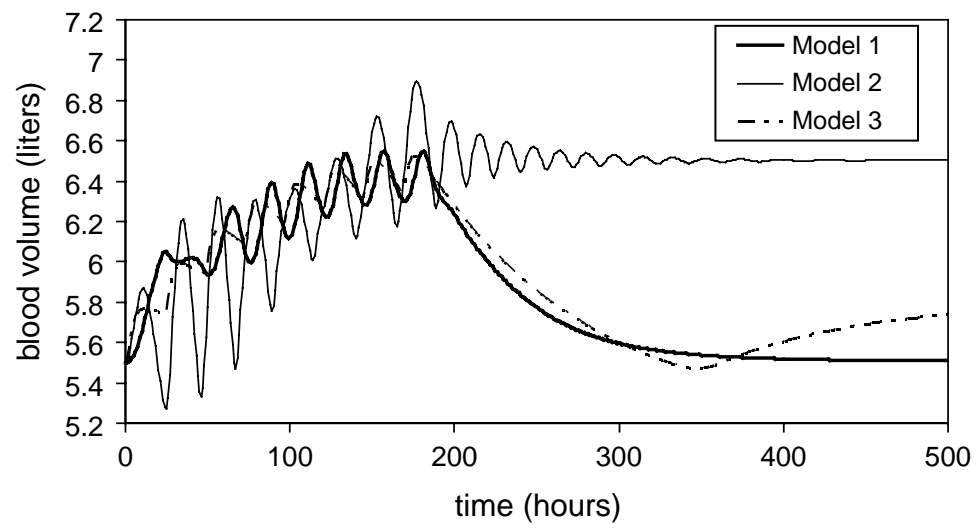

Figure 2. BVE dynamics of alternative models are not similar following calibration with experimental data from IL-11 therapy. The three models were calibrated with a clinical data set [11] and simulated under the control treatment schedule (table 2, treatment 0 ). 

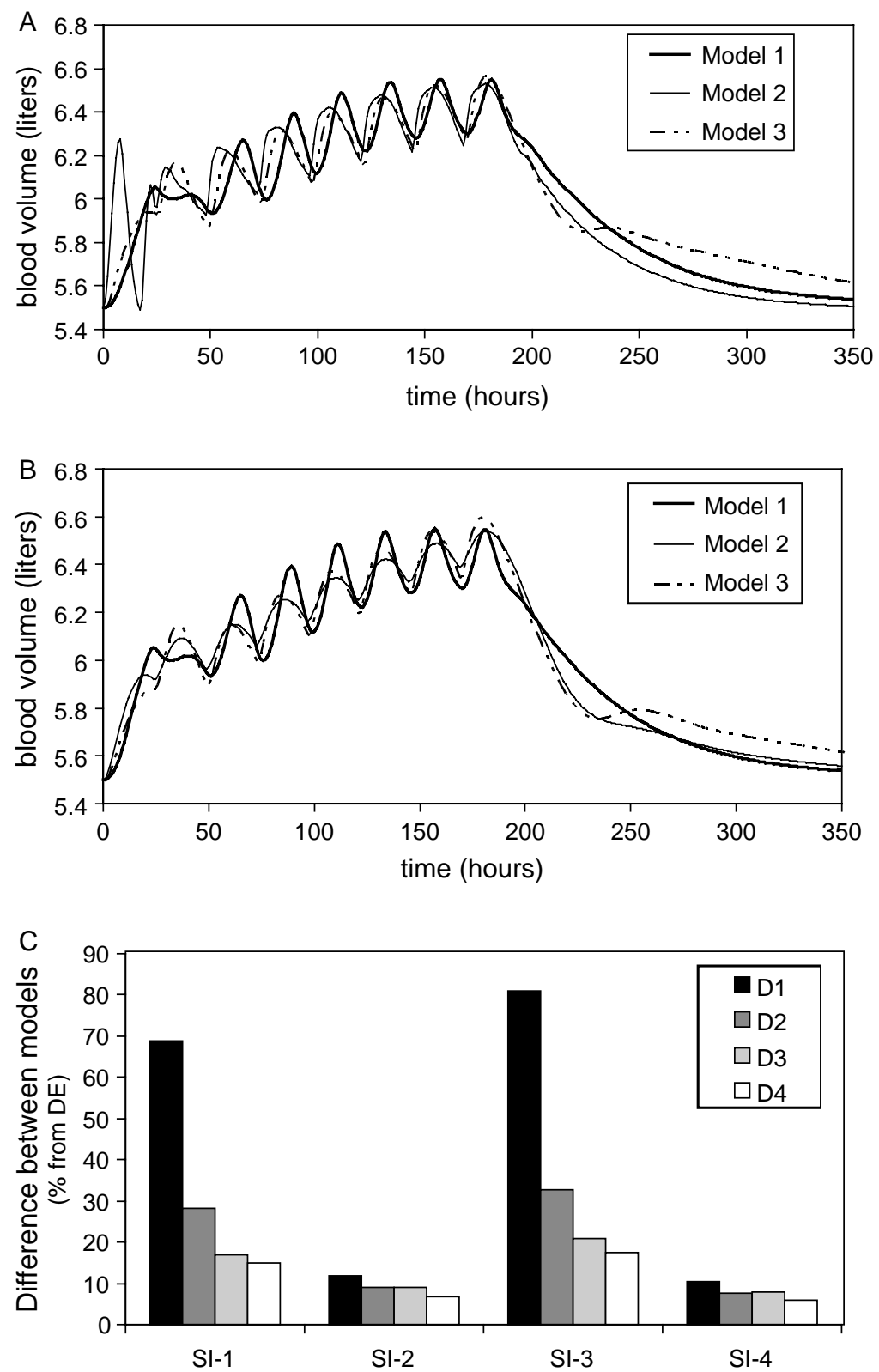

Figure 3. BVE dynamics of alternative models comply following calibration with additional data. Models 1,2 and 3 were simulated under the experimental schedule (treatment 0 ), after calibration with data sets $D 1$ (A) or $D 4$ (B), which were larger than the experimental data. A quantitative estimate of the decreased differences between the models after this calibration is shown by their similarity indexes (SI-1, SI-2, SI-3, SI-4) computed as in Ref. [24] (C). Values are relative to SI-1-4 computed following initial experimentally-based calibration.

Next, the predictability of the models was evaluated in a wider therapeutic spectrum. Treatment strategies of IL-11 (table 2), were developed according to several categories with respect to control therapy. All three models were simulated under each treatment and predictability grades were assigned to each treatment schedule (table 2) according to its SI 
values, as described in Ref. [24]. Treatments 1 and 2, similar in inter-dosing intervals and average dose intensity to the control treatment, but prolonged to 30 days, resulted in high predictability. Likewise, the high frequency treatment 4 , low frequency treatment 8 and randomly distributed treatment 10 achieved the highest predictability grades. The rest of the treatments, however, were not reliable for clinical conclusions, as observed in their lower grades (table 2 , treatments $0-2,4,8,10$ ). Collectively, the ability of the multiple models to arrive at similar outputs and thus be reliable for predictions, was highest (grade D) when the average IL-11 dose per day of the tested treatment schedule and of the control treatment (0), were equal or very similar. Accordingly, in this case, we can trust the model's BVE predictions, when simulated under treatments that comply with the therapeutic range of $\sim 25 \pm 5 \mu \mathrm{g} / \mathrm{kg} / \mathrm{day}$, independently of their duration, dosing interval, or single-injection dosage.

Subsequently, we compared the outputs of a few of the grade D (highly predictable) IL-11administering schedules (table 2 , treatments $0,2,4,8$ ), in order to qualitatively assess their safety. As expected, the high frequency treatment was the most efficacious in yielding minimal deviations in blood volume and relaxing its IL-11-induced oscillatory behaviour (figure 4). Finally, the question arose whether the models behaved similarly even for other modeled properties. The lack of similarity between the three models evaluated blood pressures even after calibration with the larger data set $D 3$ (figure 5) proved otherwise. Thus, in this work, the single predictable quantity is blood volume and conclusions regarding other model observables cannot be made.

\section{Discussion}

The anti-inflammatory and hematopoietic functions of IL-11 continue to motivate its inclusion into the arsenal of therapeutic drugs utilized in inflammatory diseases and certain cancers. Evaluating IL-11 treatment strategies and analyzing its efficacious administration can be aided by mathematical modelling, thus replacing many of the experimental trials.

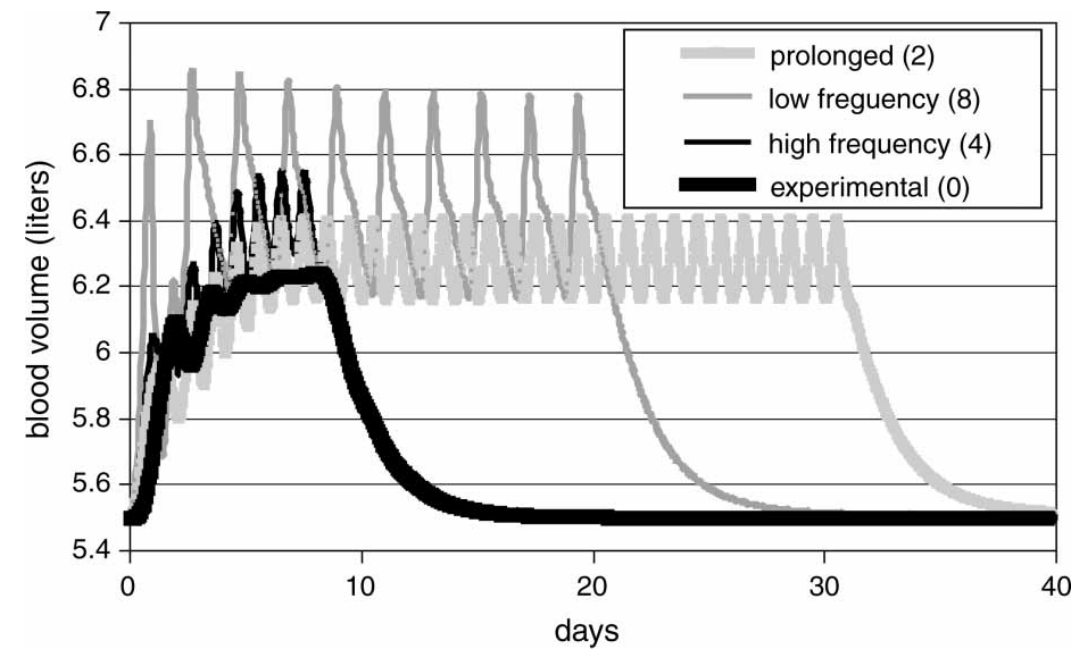

Figure 4. Simulation of highly predictable treatment scenarios. Blood volume deviations of grade D classified therapeutic schedules, simulated by model 1 following calibration with incremented data (D3), are indicated in liters. Numbers indicate treatment no. in conjunction with table 2. 


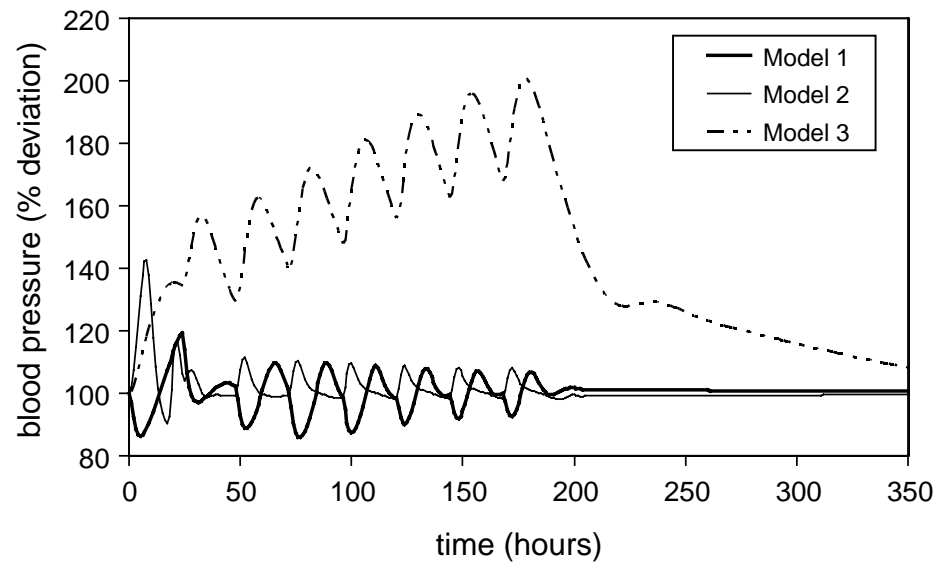

Figure 5. Blood pressure dynamics of alternative models under experimental IL-11 therapy, following refined calibration. Models were calibrated with an incremented data set $D 1$. Blood pressure dynamics were derived from model simulations and are shown as deviations from steady state values.

However, without biological knowledge of the process at hand, as well as sufficient preclinical or clinical information, the reliability of the model conclusions remains questionable. As a result, the clinically relevant but un-characterized IL-11 fluid retentive effects cannot be dealt with through conventional biomathematical methods. In an attempt to overcome these limitations, we evaluated the IL-11-induced BVE in this study, through a new multi-modelling technique. Three diverse models were constructed and their outputs were compared in order to evaluate their ability to arrive at similar BVE dynamics. We show that using this method, inconclusive knowledge of the underlying mechanism and minimal but informative data suffice to predict BVE dynamics within a relatively large spectrum of IL-11 treatments.

A few possible mechanisms have been suggested to facilitate fluid accumulation in IL-11 therapy, though none have been confirmed [11]. Preliminary studies with simple IL-11-induced BVE models, describing auto-regulated blood volume directly influenced by IL-11, could not reproduce the effects observed in clinical experiments [24]. In contrast, the models in this work, incorporating into the mechanism pressure-related changes, vascular contractility and hormonal modulators, were each capable of retrieving these experimental results. This implies that these components of the system are crucial for recreating body water stabilizing mechanisms and cannot be overridden. Moreover, as the objective of the study was to enable prediction of blood volume dynamics, rather than define the cellular and molecular mechanisms at the heart of the $\mathrm{BVE}$ effects, this macroscopic modelling in the scale of the systemic, whole-organism processes was sufficient.

Importantly, the prediction ability of the models was enabled only following their calibration with clinical measurements from various therapeutic stages, notably the stage at which blood volume levels resume steady state. It can be concluded that, given enough experimental data, the water retention effects associated with IL-11 can be retrieved even under uncertainty regarding the biological pathways. Thus, to allow predictability within a wide range of treatments, more data reflecting different time scales is needed for good predictions.

Our conclusions were confined to volume levels only, as the data for their fitting was of BVE. This limitation does not indicate an irrelevance of the models, as previously explained in Ref. [24]. It was also observed that the range of treatment regimens under which the models predictions were trustworthy, are those with average doses similar to the treatment schedule 
of the data-providing experiment. Accordingly, in this study the models outputs of BVE dynamics are reliable in IL-11 therapeutic schedules of roughly $25 \pm 5 \mu \mathrm{g} / \mathrm{kg} /$ day and of any duration. Considering the standard IL-11 levels administered (10-100 $\mu \mathrm{g} / \mathrm{kg})$ [10], it would be advisable to initiate clinical studies evaluating BVE within concentrations of 10, 20, $30, \ldots, 90 \mu \mathrm{g} / \mathrm{kg} / \mathrm{day}$. This would allow predictability of BVE within all treatment scenarios, despite obscurity of the underlying mechanism. Moreover, by collecting data of other measurable quantities, such as blood pressure and plasma hormone concentrations, the models predictability can be expanded to these properties, as well.

Within the discussed limitations, our models predict that the therapeutic schedule of low dose and high frequency administrations, equivalent to standard $25 \mu \mathrm{g} / \mathrm{kg} / \mathrm{day} \mathrm{IL}-11$, are favored over the conventional daily injections. Indeed, preclinical application of the recently suggested oral IL-11 delivery in intestinal inflammation, designed in this manner for targeting the gastrointestinal system, did result in low systemic bioavailability and increased safety [9]. Thus, development of slow-delivery and non-invasive systems for administering this drug and its analogs, such as transdermal and capsule-mediated application, may prove useful for prevention of the systemic fluid-retentive effects.

\section{Acknowledgements}

We thank Yuri Kogan and Dr. Hila Harpak for helpful insights and the Chai Foundation for support.

\section{References}

[1] Ezaki, K., 1996, Cytokine therapy for hematological malignancies, International Journal of Hematology, 65(1), 17-29.

[2] Aulitzky, W.E., Huber, C. and Peschel, C., 1993, Cytokine therapy of neoplastic and inflammatory disease, International Archives of Allergy and Immunology, 101(3), 221-226.

[3] Margolin, K.A., 2000, Interleukin-2 in the treatment of renal cancer, Seminars in Oncology, 27(2), 194-203.

[4] Vial, T. and Descotes, J., 1995, Immune-mediated side-effects of cytokines in humans, Toxicology, 105(1), $31-57$.

[5] Schwertschlag, U.S., Trepicchio, W.L., Dykstra, K.H., et al., 1999, Hematopoietic, immunomodulatory and epithelial effects of interleukin-11, Leukemia, 13(9), 1307-1315.

[6] Tepler, I., Elias, L., Smith, J.W. II, et al., 1996, A randomized placebo-controlled trial of recombinant human interleukin-11 in cancer patients with severe thrombocytopenia due to chemotherapy, Blood, 87(9), 3607-3614.

[7] Gordon, M.S., McCaskill-Stevens, W.J. Battiato, L.A., et al., 1996, A trial of recombinant human interleukin11 (neumega rhIL-11 growth factor) in women with breast cancer receiving chemotherapy, Blood, 1;87(9), $3615-3624$.

[8] Ghalib, R., Levine, C., Hassan, M., McClelland, T., et al., 2003, Recombinant human interleukin-11 improves thrombocytopenia in patients with cirrhosis, Hepatology, 37(5), 1165-1171.

[9] Cotreau, M.M., Stonis, L., Strahs, A., et al., 2004, A multiple-dose, safety, tolerability, pharmacokinetics and pharmacodynamic study of oral recombinant human interleukin-11 (oprelvekin), Biopharmaceutics and Drug Disposition, 25(7), 291-296.

[10] Smith, J.W., 2000, Tolerability and side-effect profile of rhIL-11, Oncology (Huntingt), 14(9 Suppl 8), 41-47.

[11] Dykstra, K.H., Rogge, H., Stone, A., et al., 2000, Mechanism and amelioration of recombinant human interleukin-11 (rhIL-11)-induced anemia in healthy subjects, Journal of Clinical Pharmacology, 40(8), $880-888$.

[12] Bussel, J.B., Mukherjee, R. and Stone, A.J., 2001, A pilot study of rhuIL-11 treatment of refractory ITP, American Journal of Hematology, 66, 172-177.

[13] Demitri, G.D., 2001, Targeted approaches for the treatment of thrombocytopenia, Oncologist, 6(Suppl 5), $15-23$.

[14] Arakelyan, L., Merbl, Y. and Agur, Z., 2005, Vessel maturation effects on tumour growth: validation of a computer model in implanted human ovarian carcinoma spheroids, European Journal of Cancer, 41(1), $159-167$. 
[15] Agur, Z. and Kheifetz, Y., 2005, Resonance and anti-resonance: from mathematical theory to clinical cancer treatment design. Handbook of Cancer Models with Applications to Cancer Screening, Cancer Treatment and Risk Assessment, In press.

[16] Haurie, C., Dale, D.C. and Mackey, M.C., 1998, Cyclical neutropenia and other periodic hematological disorders: a review of mechanisms and mathematical models, Blood, 92(8), 2629-2640.

[17] Skomorovski, K., Harpak, H., Ianovski, A., et al., 2003, New TPO treatment schedules of increased safety and efficacy: pre-clinical validation of a thrombopoiesis simulation model, British Journal of Haematology, 123(4), 683-691.

[18] De Pillis, L.G., Radunskaya, A.E. and Wiseman, C.L., 2005, A validated mathematical model of cell-mediated immune response to tumor growth, Cancer Research, 65(17), 7950-7958.

[19] De Pillis, L.G., Gu, W. and Radunskaya, A.E., 2005, Mixed immunotherapy and chemotherapy of tumors: modeling, applications and biological interpretations, Journal of Theoretical Biology, Epub Sep 6.

[20] Cappuccio, A., Elishmereni, M. and Agur, Z., 2006, Cancer immunotherapy by interleukin-21: potential treatment strategies evaluated in a mathematical model, Cancer Research, 66(14).

[21] Xu, J., Ren, J.F., Mugelli, A., et al., 2002, Age-dependent atrial remodeling induced by recombinant human interleukin-11: implications for atrial flutter/fibrillation, Journal of Cardiovascular Pharmacology, 39(3), 435-440.

[22] Antunes-Rodrigues, J., de Castro, M., Elias, L.L., et. al., 2004, Neuroendocrine control of body fluid metabolism, Physiological Review, 84(1), 169-208.

[23] Porth, CM, 2005, Pathophysiology, 7th ed. (Philadelphia, PA: Lippincott Williams \& Wilkins).

[24] Kheifetz, Y., Elishmereni, M., Horowitz, S., Agur, Z., 2006, In process. A new method for validating biomathematical models based on limited data: application to the thrombocytopenia-alleviating drug IL-11

[25] Katz, A.M., 2005, Physiology of the Heart, 4th ed. (Lippincott Williams \& Wilkins).

[26] Uchida, T., Aoyama, K., Mori, K., et al., 1998, Pharmacokinetics of [125I]-recombinant human interleukin-11: 1. Absorption, distribution and excretion after subcutaneous administration to male rats., European Journal of Drug Metabolism and Pharmacokinetics, 23(3), 403-410. 


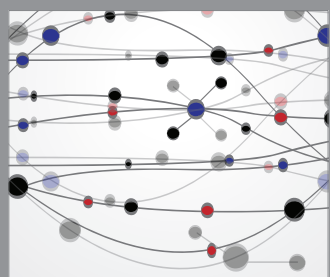

The Scientific World Journal
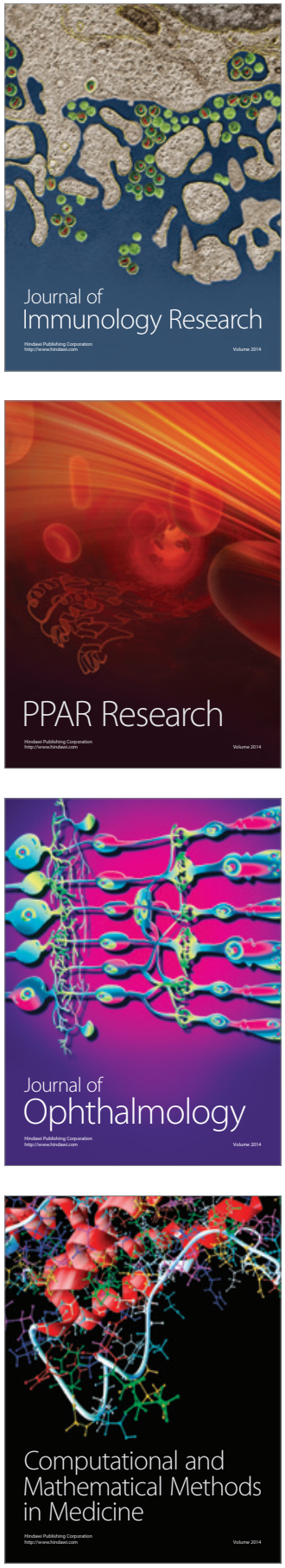

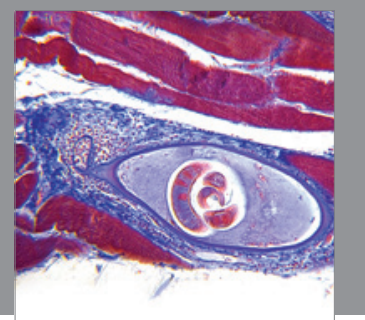

Gastroenterology

Research and Practice
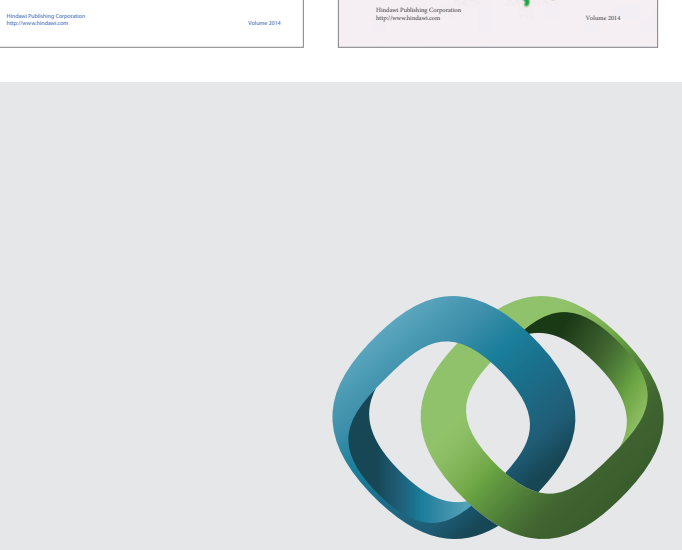

\section{Hindawi}

Submit your manuscripts at

http://www.hindawi.com
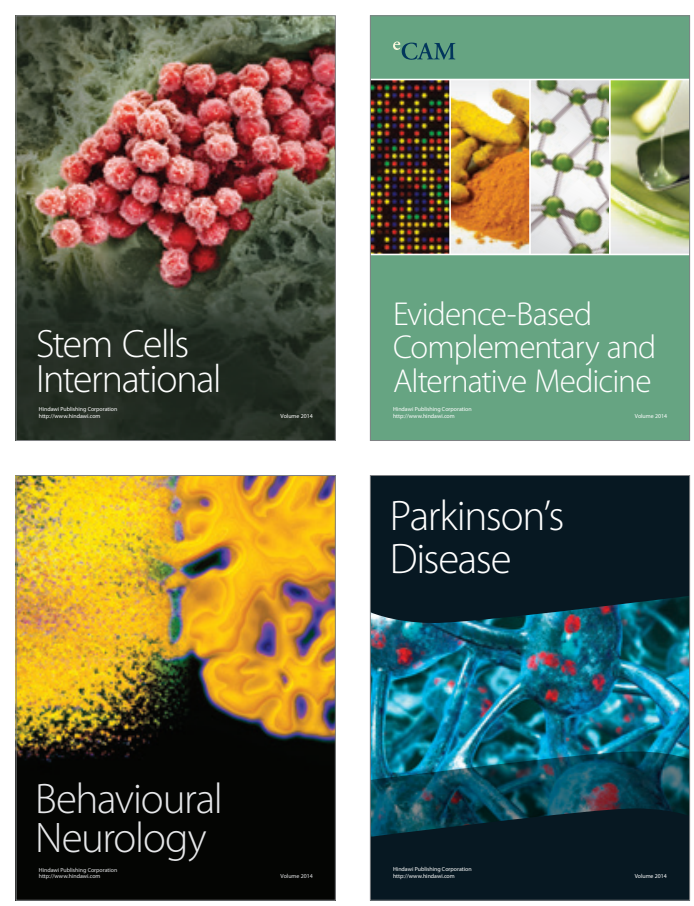

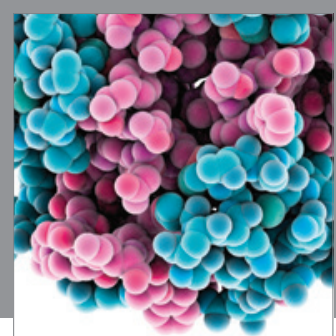

Journal of
Diabetes Research

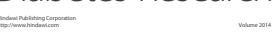

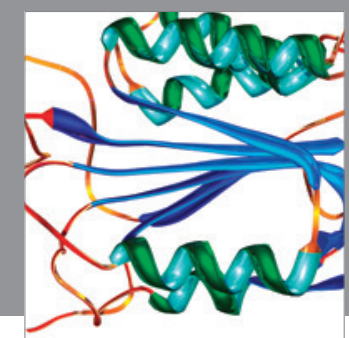

Disease Markers
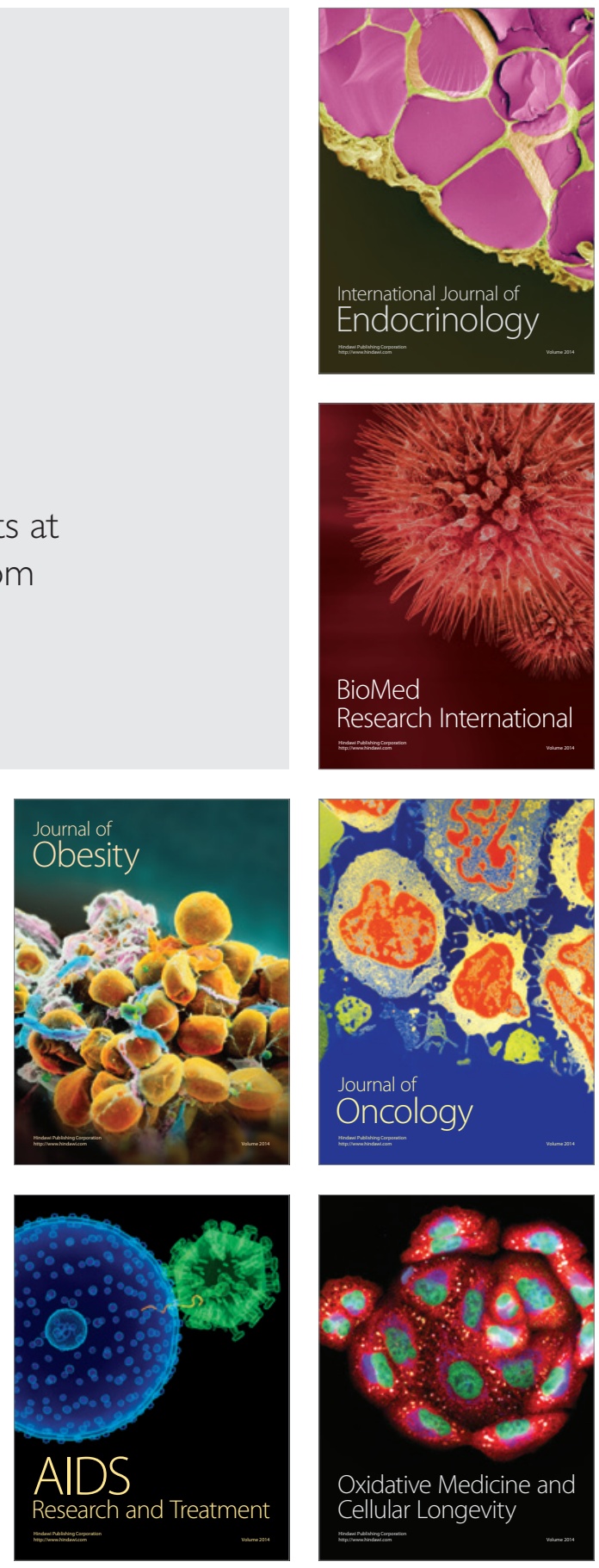